\title{
Sporulation environment drives phenotypic variation in the pathogen Aspergillus fumigatus
}

\author{
S. Earl Kang, ${ }^{1, \dagger}$ Brandi N. Celia (DD, ${ }^{1}$ Douda Bensasson (D) ${ }^{2}$ and Michelle Momany (D) ${ }^{1, *}$ \\ ${ }^{1}$ Fungal Biology Group \& Plant Biology Department, University of Georgia, Athens, GA 30602, USA \\ ${ }^{2}$ Institute of Bioinformatics \& Plant Biology Department, University of Georgia, Athens, GA 30602, USA \\ *Corresponding author: University of Georgia, 2502 Miller Plant Sciences, Athens, GA 30602, USA. Email: mmomany@uga.edu \\ ${ }^{\dagger}$ Present address: Fungal Team, Ginkgo Bioworks, Boston, MA 02210, USA.
}

\begin{abstract}
Aspergillus fumigatus causes more than 300,000 life-threatening infections annually and is widespread across varied environments with a single colony producing thousands of conidia, genetically identical dormant spores. Conidia are easily wind-dispersed to new environments where they can germinate and, if inhaled by susceptible hosts, cause disease. Using high-throughput single-cell analysis via flow cytometry we analyzed conidia produced and germinated in nine environmentally and medically relevant conditions (complete medium, minimal medium, high temperature, excess copper, excess iron, limited iron, excess salt, excess reactive oxygen species, and limited zinc). We found that germination phenotypes vary among genetically identical individuals, that the environment of spore production determines the size of spores and the degree of germination heterogeneity, and that the environment of spore production impacts virulence in a Galleria mellonella host.
\end{abstract}

Keywords: sporulation; conidia; heterogenetity; flow cytometry

\section{Introduction}

Fungal diseases kill over 1.5 million people each year (Brown et al. 2012; Bongomin et al. 2017). Rather than spreading patient-topatient, fungal diseases are acquired from the environment or host normal flora. Nine of the ten most common agents of fungal disease can be spread via spores (Botts and Hull 2010; Brown et al. 2012). Breaking dormancy, or germinating, is arguably the most important step in pathogenesis for these fungi. Historically studies have focused on the germination environment, addressing factors such as temperature, inoculum density, carbon source, nitrogen source, and pH (Brown 1922; Loo 1976; Osherov and May 2001; Araujo and Rodrigues 2004; Wang et al. 2019). However, despite the wide range of environments in which fungal spores are produced and their importance as disease agents, the impact of sporulation environment on germination has been largely ignored. To determine whether the environment of sporulation influences germination, we performed sporulation/germination swap experiments in which spores of the fungal pathogen Aspergillus fumigatus were produced in one of nine environmentally or medically relevant conditions and then shifted to one of the same nine conditions for germination. By monitoring hundreds of thousands of spores, we found that germination phenotypes vary among genetically identical individuals and that the environment of spore production determines the size of spores and the degree of germination heterogeneity. By testing the ability of spores produced in different environments to kill a G. mellonella host, we also found that sporulation environment impacts virulence.

\section{Materials and methods Fungal strains, cultivation, and preparation of conidia}

A. fumigatus CEA10 was cultivated on $1.5 \%$ agar solid complete medium (CM) or minimal medium (MM) as previously described (Momany et al. 1999) with modifications as described in Table 1. For conidial stock preparation, conidia were produced on complete media, harvested in sterile water, and $1 \times 10^{6}$ conidia in $500 \mu \mathrm{l}$ of ddH2O were plated in a homogenous layer on $25 \mathrm{ml}$ of solid 1\% glucose Aspergillus MM with modifications described in Table 1 in $90 \mathrm{~mm}$ plates in 3 technical replicates. Plates were incubated in the dark, stored upside down at $37^{\circ} \mathrm{C}$ or $50^{\circ} \mathrm{C}$ for 72 hours. A. fumigatus conidia from 3 plates were harvested by overlaying plates with $25 \mathrm{ml}$ sterile ddH2O, combining conidia and filtering through 22-25 $\mu \mathrm{m}$ Miracloth (MilliporeSigma, St. Louis, MO, USA). Conidia were washed twice in ddH2O and counted using a hemocytometer.

\section{Germination assay}

Conidia from 3 plates were pooled and identical aliquots of 3-5 $\times$ $10^{5} \mathrm{C} / \mathrm{ml}$ were added to liquid germination conditions described in Table 1 (Araujo and Rodrigues 2004). Cultures were incubated for 6 hours at $37^{\circ} \mathrm{C}$ or $50^{\circ} \mathrm{C} @ 250 \mathrm{rpm}$ in dark, then fixed with $2.5 \%$ formaldehyde. Eighty-one conditions were analyzed in total. Controls included conidia fixed at 0 hour in liquid germination conditions. 
Table 1 Sporulation and germination conditions

\begin{tabular}{|c|c|c|c|}
\hline Abbreviation & Description & Medium & Temperature $\left({ }^{\circ} \mathrm{C}\right)$ \\
\hline $\mathrm{CM}$ & Complete medium & $\begin{array}{l}\text { Nutrient-rich undefined medium containing yeast } \\
\text { extract, glucose, nitrogen, and vitamins }\end{array}$ & 37 \\
\hline MM & Minimal medium & $\begin{array}{l}\text { Nutrient-rich defined synthetic medium } \\
\text { containing glucose, nitrogen, and vitamins }\end{array}$ & 37 \\
\hline $50^{\circ} \mathrm{C}$ & High temperature stress & MM & 50 \\
\hline$+\mathrm{Cu}$ & Copper stress & MM with $1 \mathrm{mM} \mathrm{CuSO}_{4}$ & 37 \\
\hline$+\mathrm{Fe}$ & Excessive iron stress & MM with $10 \mathrm{mM} \mathrm{FeSO}_{4}$ & 37 \\
\hline$-\mathrm{Fe}$ & Iron limiting stress & MM without $\mathrm{FeSO}_{4}$ & 37 \\
\hline $\mathrm{NaCl}$ & Osmotic or salt stress & MM with $0.5 \mathrm{M} \mathrm{NaCl}$ & 37 \\
\hline $\mathrm{H}_{2} \mathrm{O}_{2}$ & Reactive oxygen species (ROS) stress & MM with $2 \mathrm{mM} \mathrm{H}_{2} \mathrm{O}_{2}$ & 37 \\
\hline$-\mathrm{Zn}$ & Zinc limiting stress & $\mathrm{MM}$ without $\mathrm{ZnSO}_{4}$ & 37 \\
\hline
\end{tabular}

\section{Analysis of germination/flow cytometry}

Flow cytometry was performed at the Center for Tropical and Emerging Global Diseases Cytometry Shared Resource Laboratory at the University of Georgia on a CyAn ADP using Summit, version 4.3 (Beckman Coulter, Fullerton, CA, USA). Between 20,000 and 250,000 events (cells) were analyzed in four replicates for each fixed pre- and post-germination sample. Due to the sensitivity of flow cytometry and small particulates in the germinated samples, forward scatter and side scatter values smaller than fixed ungerminated conidia were filtered from the analysis. FlowJo flow cytometry analysis software, version 10 (Tree Star, Ashland, OR, USA) was used for analysis and histogram. Histogram represents the linear scaled forward scatter data to better visualize the variation in germination. Conidial morphologies (including the absence of significant clumping) were verified by microscopic observation for all experiments and by Amnis ImageStream (Amnis MerckMillipore Sigma, Seattle, WA, USA) for three experiments.

\section{Statistical analysis of germination}

Forward scatter scaled linear or log data were combined for each condition from all replicates. Linear and log data were checked for normality using D'Agostino-Pearson test (D'Agostino et al. 1990). Due to nonparametric distribution, comparison between multiple groups were analyzed by Kruskal-Wallis test followed by one-sided Dunn's multiple comparison test(Dunn 1964) using GraphPad Prism version 8 (GraphPad Software, La Jolla, CA, USA). Robust coefficient of variance ( $\mathrm{rCV}$ ) was calculated using $100 * 1 /$ 2 (Intensity [at 84.13 percentile]-Intensity [at 15.87 percentile])/ Median using FlowJo v10 (Tree Star, Ashland, OR, USA). Pearson correlation analysis followed by a two-tailed test was performed to assess the relationship between median log forward scatter (growth) and rCV (variation) in a given germination condition using GraphPad Prism version 8 (GraphPad Software, La Jolla, CA, USA).

\section{Viability assay_live/dead staining}

For viability assays, two replicates of unfixed cells (conidia and germlings) were co-stained with $10 \mu \mathrm{g} / \mathrm{ml}$ fluorescein diacetate (FDA) and $2 \mu \mathrm{g} / \mathrm{ml}$ propidium iodine (PI) for 5 minutes in the dark, then 20,000 events were analyzed immediately using flow cytometry to measure size (forward scatter) and fluorescence. Controls included unstained and FDA, PI, and FDA+PI stained live and dead (ethanol-killed) cells.

\section{Galleria mellonella infections}

G. mellonella larvae (waxworms.net, St. Marys, OH, USA) were stored in wood shavings in the dark at room temperature prior to use. Wax-moths in the final instar larval stage were used for injections using the method of Jackson et al (Jackson et al. 2009). Briefly G. mellonella was placed at $20^{\circ} \mathrm{C}$ for 25 minutes and placed on ice prior to injections. Using a cotton swab dipped in ethanol, G. mellonella prolegs were cleaned and the left leg of the 3rd set of prolegs via the hemocoel were injected with a total of $10^{6}$ conidia in $5 \mu$ l of PBS solution using a Hamilton syringe. Fifteen larvae were infected for each condition. After injections, larvae were incubated in petri plates at $37^{\circ} \mathrm{C}$ for 72 hours with observation every 12 hours. G. mellonella were considered dead if they had no response to touch. G. mellonella larvae were shipped to the lab in separate batches. Five replicate experiments were performed. No injection and PBS only controls were included for all replicates. G. mellonella was infected with conidia that had been produced under five different conditions: $\mathrm{MM}, 50^{\circ} \mathrm{C},+\mathrm{Fe}, \mathrm{NaCl}$, and $-\mathrm{Zn}$. Fifteen larvae were injected with each conidium type in 5 separate replicates, so in total 375 larvae were injected.

\section{Statistical analysis of infection assays}

To test for differences among the batches of larvae and between the 5 sporulation conditions on host survival, we fit a generalized linear mixed-effects model (GLMM) using R (version 4.0.2) and the glmer function from the lme4 package (version 1.1-26; Bates et al. 2020). For this survival analysis, the time last seen alive in hours (Time) was set as the response variable. We used the gamma family of errors because variance increases with increasing mean host age at death (Crawley 2012). Replicates were included in the model as a random effect, and we tested for differences among conidia exposed to 5 different sporulation Treatments: MM, 50 C, Fe, $\mathrm{NaCl}$ and Zn; Time $\sim$ Treatments $+(1 \mid$ Replicates). The model was fit with default settings for glmer; with maximum likelihood using the Laplace approximation. We tested for differences among treatments through a likelihood ratio test on nested models using the drop1 function with the "Chisq" option, and P-values in Table 3 are based on the default asymptotic Wald tests for glmer models (Bates et al. 2020). As a control, we repeated the above analysis using a mixed-effects Cox model implemented in $\mathrm{R}$ with coxme (version 2.2-16). This second analysis confirms the significant differences among replicates (Supplementary Figure S3; Likelihood ratio test, $d f=1$, $\mathrm{P}=2 \times 10^{-10}$ ) and among treatments of conidia (Table 3; Likelihood ratio test, $\mathrm{d} f=4, \mathrm{P}=0.003$ ). The data table and $\mathrm{R}$ script used for analyses are available at https://github.com/bensasson lab/data/tree/master/kang_etal20.

\section{Data availability}

Strains and plasmids are available upon request. The authors affirm that all data necessary for confirming the conclusions of the 
article are present within the article, figures, and tables. Supplemental material available at G3 online.

\section{Results and discussion}

We hypothesized that exposure to specific stresses during sporulation might lead to better germination in the same or related conditions. To test this hypothesis, we performed single-cell experiments in which A. fumigatus was sporulated under nine environmentally and medically relevant conditions (Errasquin et al. 2002; Tepsic et al. 2006; Haas 2012; Amich and Calera 2014) and the resulting conidia were transferred to all nine conditions for germination (Table 1). To avoid induction or selection of mutations during sporulation, we did not use serial passaging; rather, identical aliquots of inoculum were incubated for 72 hours on nine types of solid medium for the production of conidia, and identical aliquots of conidia from each condition were transferred directly to nine types of liquid medium for germination (Supplementary Figure S1).

After 6 hours incubation we used flow cytometry to detect any increase in cell size, a clear indication that germination had been initiated. The entire 9 by 9 sporulation/germination swap experiment was repeated four times. We recorded forward scatter for approximately 20,000 conidia and germlings for each condition in each replicate. For each condition, data from all replicates were concatenated and analyzed as a single population (Figure 1 and Table 2).

Dormant conidia produced in all sporulation environments showed very similar forward scatter profiles except for conidia produced at $50^{\circ} \mathrm{C}$, in which the forward scatter peak shifted slightly to the right, suggesting a larger size (red peaks in Figure 1). Microscopic examination showed that conidia produced at $37^{\circ} \mathrm{C}$ were approximately $2-3 \mu \mathrm{m}$ in diameter, while those produced at $50^{\circ} \mathrm{C}$ were approximately 1.5 times larger (Supplementary Figure S2).

Not surprisingly, the rate at which conidia broke dormancy and grew varied depending on germination conditions. Conidia germinated in standard media containing sufficient metals (CM, $\mathrm{MM})$ at optimal temperature $\left(37^{\circ} \mathrm{C}\right)$ showed larger median forward scatter values indicating faster growth compared to conidia germinated in media with metal limitation ( $\mathrm{Zn}$ and $-\mathrm{Fe})$, at elevated temperature $\left(50^{\circ} \mathrm{C}\right)$, or subjected to stressors $(+\mathrm{Cu},+\mathrm{Fe}$, $\mathrm{NaCl}$, and $\mathrm{H}_{2} \mathrm{O}_{2}$ ) (blue peaks in Figure 1 and Supplementary Table S1). Conidia from all sporulation environments broke dormancy and grew more quickly in CM germination medium than in any other germination condition. Conidia germinated in $0.5 \mathrm{M} \mathrm{NaCl}$ (osmotic stress) generally broke dormancy and grew more slowly than those in other germination conditions. These results are consistent with previous work showing that rich medium and nonstressful conditions during germination favor more rapid dormancy breaking and growth (Schmit and Brody 1976; Meletiadis 2001; Osherov 2009).

In addition to the expected contribution of germination conditions, the rate at which conidia broke dormancy and grew varied depending on sporulation environment. The sporulation environments that favored rapid dormancy breaking and growth were not the same as the germination conditions that favored it (blue peaks in Figure 1 and Supplementary Table S2). As discussed above, $0.5 \mathrm{M} \mathrm{NaCl}$ during germination resulted in reduced dormancy breaking and growth as indicated by reduced median forward scatter (Supplementary Table S1). In contrast, osmotic stress imposed by $0.5 \mathrm{M} \mathrm{NaCl}$ during sporulation resulted in conidia that broke dormancy and grew more quickly across germination conditions as indicated by increased median forward scatter (Supplementary Table S2). In addition to $\mathrm{NaCl}$ medium, sporulation on MM or +Fe medium generally improved dormancy breaking and growth when compared to conidia from all other sporulation environments. Conidia from $+\mathrm{Cu},-\mathrm{Fe}$, and $-\mathrm{Zn}$ sporulation environments generally performed worse when compared to conidia from the MM condition suggesting that proper metal homeostasis is necessary during sporulation as well as germination. These results show for the first time that sporulation environment impacts the ability of a medically important fungus to break dormancy and grow across multiple germination environments.

While we predicted that forward scatter peaks might shift left or right with changes in germination or sporulation conditions, we were surprised to see striking differences in the widths and shapes of peaks depending on sporulation environment (blue peaks in Figure 1). A. fumigatus conidia are clonal, with each conidium in a colony containing a single genetically identical nucleus produced by mitosis. Previous work has shown that conidia remain dormant until they are exposed to a carbon source and water (Osherov and May 2001), at which time individuals in the population synchronously break dormancy and start growth, with rough synchrony maintained through at least the first 12 hours (Momany and Taylor 2000). Thus, we expected that individual conidia produced in the same sporulation environment would break dormancy and grow synchronously, giving rise to relatively narrow peaks. The observed wide peaks show that genetically identical conidia within the same population break dormancy and grow at different rates. The dramatic leftward shift of post-germination peaks for sporulation conditions such as - Zn medium could be explained if $\mathrm{Zn}$ deficiency during sporulation killed conidia. However, viability assays with FDA and propidium iodide showed that conidia sporulated on MM and on - Zn media contained very similar, low numbers of propidium iodide stained cells and that most of the conidia that did not enlarge during germination were not dead (Supplementary Table S3).

To better understand the range of individual variation within genetically identical clonal populations of conidia, we compared the robust coefficient of variation ( $\mathrm{rCV}$, the normalized standard deviation of the median) for forward scatter of each sporulation/ germination pair (Table 2 and Supplementary Table S2). Conidia that were produced on $\mathrm{NaCl},+\mathrm{Fe}, 50^{\circ} \mathrm{C}$, and $\mathrm{MM}$ sporulation media showed lower rCV values and narrower forward scatter peaks across germination conditions, indicating less size variation among individuals in those populations. Conidia from $-\mathrm{Zn},-\mathrm{Fe}$, $\mathrm{CM},+\mathrm{Cu}$, and $\mathrm{H}_{2} \mathrm{O}_{2}$ sporulation medium showed higher $\mathrm{rCV}$ values and wider forward scatter peaks across germination conditions, indicating more variation in germling size (Figure 1 and Supplementary Table S2). Taken together with median forward scatter values this shows that conidia that germinate faster tend to germinate more synchronously. Indeed, there was a negative correlation between germling size (median forward scatter) and size variation (rCV) across most conditions (Table 2). The correlation between germling size and its variation was stronger when comparing sporulation environments $\left(r^{2}=0.9, P<0.0001\right.$; Supplementary Table S2) than when comparing germination conditions $\left(r^{2}=0.6, P=0.02\right.$; Supplementary Table S1) consistent with the idea that the environment of sporulation drives germination variation.

To determine whether the environment of sporulation has an impact on the pathogenicity of A. fumigatus, we took advantage of a G. mellonella (wax moth) infection system (Jackson et al. 2009). Fifteen G. mellonella larvae were injected with a PBS control or $10^{6}$ 


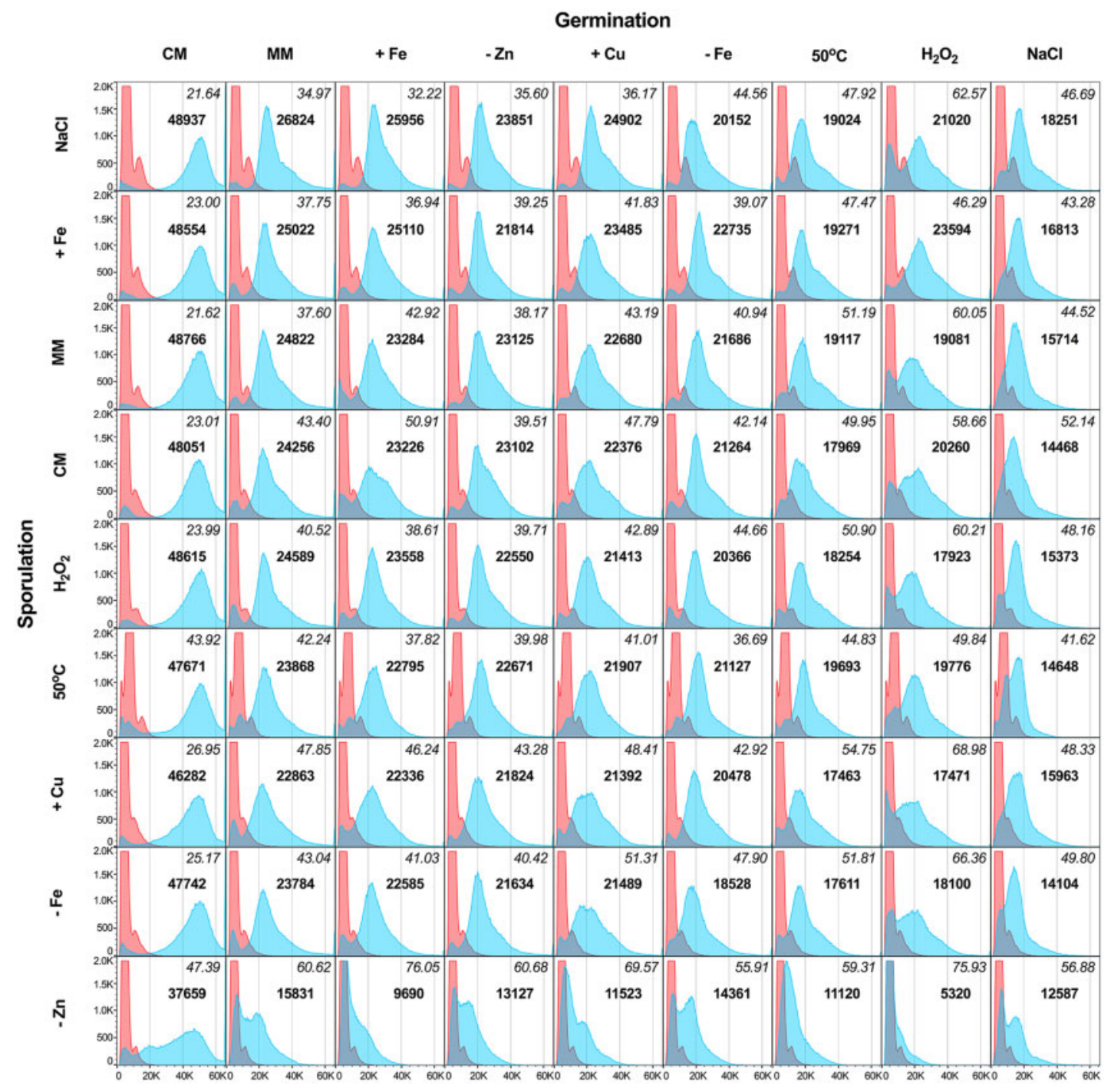

Figure 1 Sporulation conditions impact germination of A. fumigatus conidia. Conidia produced in one of nine sporulation environments were transferred to all nine conditions for germination. The $x$-axis shows the linear forward scatter, an indication of relative size. The $y$-axis shows the number of events (cells) counted. Red peaks show forward scatter of dormant conidia from each sporulation condition measured before germination. Blue peaks show forward scatter after 6 hours incubation in each germination condition. Bold values are the median of linear forward scatter values after germination. Italicized values are the robust coefficient of variation (normalized standard deviation around the median) of linear forward scatter values after germination.

A. fumigatus dormant conidia produced on $\mathrm{MM}, 50^{\circ} \mathrm{C},+\mathrm{Fe}, \mathrm{NaCl}$, or $-\mathrm{Zn}$ sporulation medium. Larvae were incubated at $37^{\circ} \mathrm{C}$ and observed every 12 hours for 3 days. Larvae that were unresponsive in a touch test were counted as dead. Five independent replicates were performed. Most G. mellonella larvae injected with PBS (control) survived to the end of the experiment (88\%) (Supplementary Figure S3F) and most G. mellonella larvae injected with conidia from any sporulation condition died by the end of the experiment (92.8\%) (Supplementary Figure S3, A-E). In many replicates, larvae injected with conidia produced under conditions that led to more rapid and synchronous germination (Supplementary Table S3, $\mathrm{MM}, 50^{\circ} \mathrm{C},+\mathrm{Fe}$, and $\mathrm{NaCl}$ ) died more quickly than those from conditions that led to slower and less synchronous germination $(-\mathrm{Zn})$ (Supplementary Figure S3, 48 hours timepoint, A-D us E). However, we observed inconsistencies in host survival time between replicates likely because live $G$. mellonella larvae were shipped to the lab in separate batches and so had random differences in past exposure to stress. To separate the effects of sporulation conditions of A. fumigatus from random host batch effects of G. mellonella larvae we fit a generalized linear mixed model (GLMM) (Crawley 2012; Bates et al. 2020) to the data from all replicates (75 larvae from each sporulation condition for a total of 375). Even after accounting for random host batch effects of replicates, we found differences in G. mellonella survival times after infection with A. fumigatus conidia produced in the five sporulation environments (Likelihood ratio test, $\chi^{2}=19.1$, $d f=4, P=0.0008)$. The most striking difference was in average survival time of $G$. mellonella injected with conidia produced at $50^{\circ} \mathrm{C}$ (22 hours) compared to those produced under other conditions (30-32 hours, $P<0.005$, Table 3 ).

It is not surprising that some sporulation conditions which strongly impacted the speed and synchrony of germination in sporulation/germination swap experiments did not impact virulence in G. mellonella infection assays. The sporulation/germination swap experiments were performed over 6 hours in uniform, defined synthetic medium (Figure 1 and Table 2) while the G. mellonella infection assays were performed over 72 hours in larvae that contain many different microenvironments and can deploy host defenses (Supplementary Figure S3 
Table 2 Statistical analysis of all sporulation/germination combinations grouped by germination condition

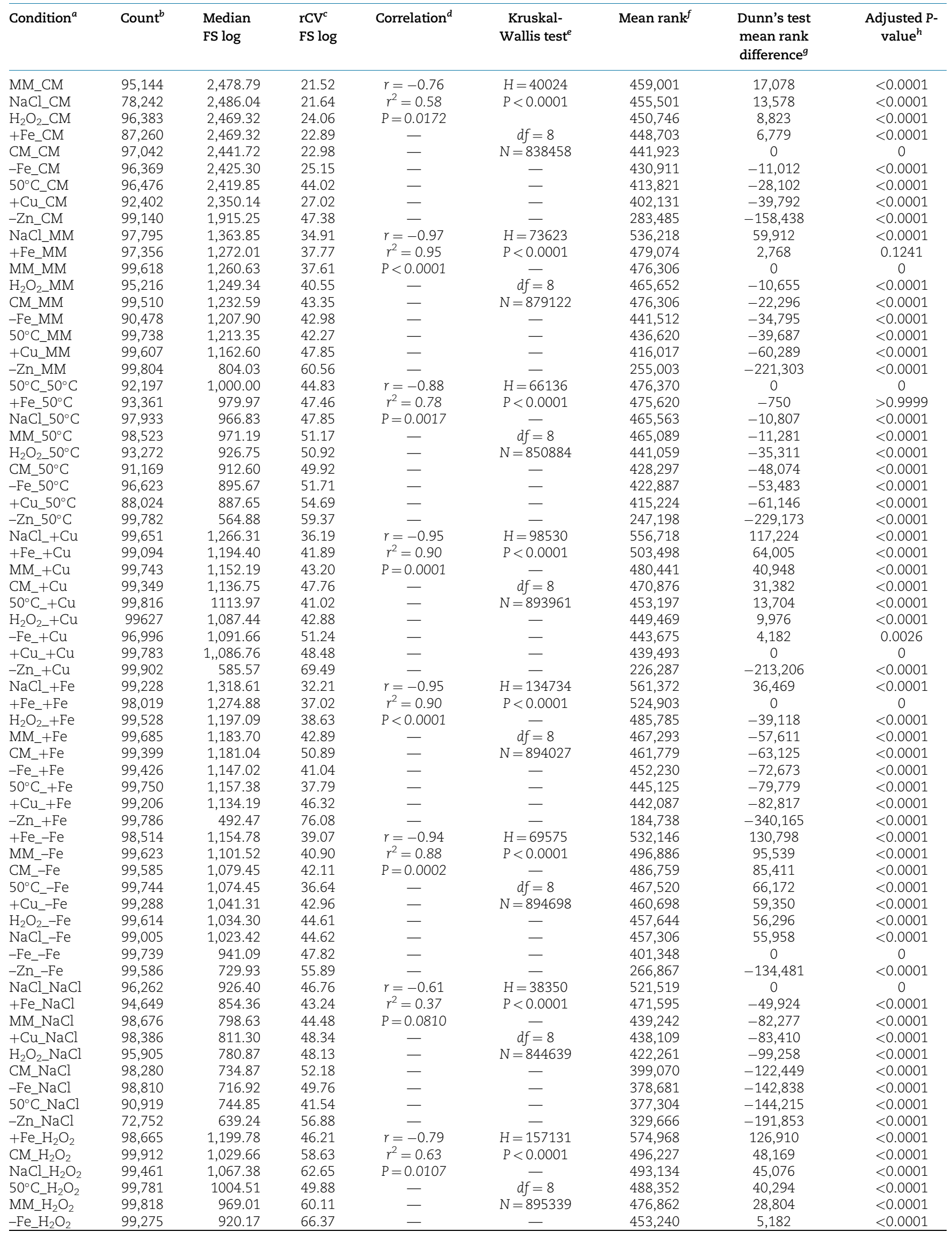


Table 2. (continued)

\begin{tabular}{|c|c|c|c|c|c|c|c|c|}
\hline Condition $^{a}$ & Count $^{b}$ & $\begin{array}{l}\text { Median } \\
\text { FS log }\end{array}$ & $\begin{array}{l}\mathrm{rCV}^{\mathrm{c}} \\
\mathrm{FS} \log \end{array}$ & Correlation $^{d}$ & $\begin{array}{c}\text { Kruskal- } \\
\text { Wallis test }^{e}\end{array}$ & Mean rank $f^{f}$ & $\begin{array}{l}\text { Dunn's test } \\
\text { mean rank } \\
\text { difference }^{g}\end{array}$ & $\begin{array}{l}\text { Adjusted P- } \\
\text { value }^{h}\end{array}$ \\
\hline $\begin{array}{l}\mathrm{H}_{2} \mathrm{O}_{2} \mathrm{H}_{2} \mathrm{O}_{2} \\
+\mathrm{Cu} \mathrm{H}_{2} \mathrm{O}_{2} \\
-\mathrm{Zn}_{\mathrm{H}} \mathrm{H}_{2} \\
\mathrm{NaCl}-\mathrm{Zn} \\
\mathrm{MM} \text { - } \mathrm{Zn} \\
\mathrm{CM}_{2}-\mathrm{Zn} \\
\mathrm{H}_{2} \mathrm{O}_{2} \text { - } \mathrm{Zn} \\
50^{\circ} \mathrm{C}_{-}-\mathrm{Zn} \\
+\mathrm{Fe}-\mathrm{Zn} \\
+\mathrm{Cu}-\mathrm{Zn} \\
-\mathrm{Fe} \text { - } \mathrm{Zn} \\
-\mathrm{Zn}-\mathrm{Zn}\end{array}$ & $\begin{array}{l}99,386 \\
99,514 \\
99,527 \\
99,601 \\
99,801 \\
99,818 \\
99,767 \\
99,821 \\
98,771 \\
99,836 \\
99,757 \\
99,909\end{array}$ & $\begin{array}{r}909.88 \\
887.65 \\
270.17 \\
1,210.62 \\
1,175.74 \\
1,173.10 \\
1,144.79 \\
1,152.19 \\
1,108.98 \\
1,108.98 \\
1,099.05 \\
667.14\end{array}$ & $\begin{array}{l}60.18 \\
69.01 \\
76.08 \\
35.68 \\
38.20 \\
39.53 \\
39.64 \\
40.04 \\
39.27 \\
43.28 \\
40.47 \\
60.62\end{array}$ & $\begin{array}{c}- \\
- \\
r=-0.99 \\
r^{2}=0.97 \\
P<0.0001 \\
- \\
- \\
- \\
- \\
-\end{array}$ & $\begin{array}{c}- \\
- \\
\mathrm{H}= \\
\mathrm{P}<097081 \\
- \\
\mathrm{df}=8 \\
\mathrm{~N}=89001 \\
- \\
- \\
- \\
-\end{array}$ & $\begin{array}{l}448,058 \\
438,229 \\
160,729 \\
525,981 \\
498,613 \\
487,019 \\
483,843 \\
470,437 \\
456,940 \\
449,778 \\
449,086 \\
215,668\end{array}$ & $\begin{array}{c}0 \\
-9,830 \\
-287,330 \\
310,313 \\
282,945 \\
271,350 \\
268,175 \\
254,769 \\
241,272 \\
234,109 \\
233,418 \\
0\end{array}$ & $\begin{array}{c}0 \\
<0.0001 \\
<0.0001 \\
<0.0001 \\
<0.0001 \\
<0.0001 \\
<0.0001 \\
<0.0001 \\
<0.0001 \\
<0.0001 \\
<0.0001 \\
0\end{array}$ \\
\hline
\end{tabular}

a Sporulation_Germination denotes conidia transferred from solid medium sporulation environment into liquid medium germination conditions as described in Table 1.

b Number of events (cells) analyzed by flow cytometry.

c $\mathrm{rCV}=$ normalized standard deviation of the median, an indication of variance in the population.

d Pearson correlation analysis between median forward scatter and observed variation (rCV) within a germination group. $r=$ correlation coefficient

e The Kruskall-Wallis test determines whether there is a difference in distribution between multiple groups and is performed on ranked data. $\mathrm{H}=$ the KruskallWallis statistic, an indication of the difference between groups; $\mathrm{df}=$ degrees of freedom. The P-values indicate the significance of differences among sporulation environments in the germination condition.

$\mathrm{f}$ Mean rank from Kruskal-Wallis test indicates which sporulation conditions tend to have the greatest values in the germination group

g Dunn's multiple comparison test. Mean rank for each sporulation environment in the same germination condition was compared to the mean rank of the same sporulation and germination conditions. Dunn's test compares the difference in the sum of ranks between two samples with the expected average difference (based on the number of the groups and size).

$\mathrm{h}$ Significance: $\left.\mathrm{P}>0.05(\mathrm{~ns}), \mathrm{P} \leq 0.05\left(^{(}\right), \mathrm{P} \leq 0.01\left(^{* *}\right), \mathrm{P} \leq 0.001{ }^{(* *}\right), \mathrm{P} \leq 0.0001\left(^{(* * *}\right)$ was determined using Dunn's test comparing the difference in the mean ranks between each sporulation condition and matching sporulation and germination conditions.

Table 3 G. mellonella estimated survival times (in hours) after injection with A. fumigatus conidia produced in differing sporulation conditions

\begin{tabular}{lccccc}
\hline & $\mathbf{5 0 ^ { \circ } \mathbf { C }}$ & $+\mathbf{F e}$ & $\mathbf{M M}$ & $\mathbf{N a C l}$ & $-\mathbf{Z n}$ \\
\hline Replicate $1(N=15)$ & 24 & 33 & 35 & 36 & 35 \\
Replicate 2 $(N=15)$ & 20 & 25 & 26 & 26 & 26 \\
Replicate 3 $(N=15)$ & 17 & 21 & 22 & 23 & 23 \\
Replicate 4 $(N=15)$ & 25 & 34 & 35 & 37 & 36 \\
Replicate $(N=15)$ & 26 & 35 & 37 & 38 & 39 \\
Average $(N=75)$ & 22 & $30^{*}$ & $31^{* *}$ & $32^{* * *}$ & $32^{* * *}$
\end{tabular}

Averages were calculated over 5 experimental replicates, and T-tests for the difference between conidia sporulated at $50^{\circ} \mathrm{C}$ and other treatments are from a generalized linear mixed model (see Materials and Methods): ${ }^{*}<<0.005$

${ }^{*} P<0.001,{ }^{* * *} P<0.00005$

and Table 3). Each sporulation/germination swap experiment measured tens of thousands of germination events, while G. mellonella infection experiments each measured 75 events. Despite the differences in these assays, it is clear that sporulation at $50^{\circ} \mathrm{C}$ had a strong impact on sporulation, germination, and virulence. Compared to conidia produced under other sporulation conditions, dormant conidia produced at $50^{\circ} \mathrm{C}$ were larger (red peaks in Figure 1 and Supplementary Figure S2), broke dormancy and grew with moderate speed and synchrony (blue peaks in Figure 1 and Supplementary Table S2), and killed G. mellonella larvae faster (Supplementary Figure S3 and Table 3). We do not know the mechanism for faster killing by conidia produced at $50^{\circ} \mathrm{C}$, but it might be especially important in agricultural settings since A. fumigatus is thermotolerant and known to grow in compost (Errasquin et al. 2002).

Our results show for the first time that the environment of spore production impacts the spore size, germination, and virulence of A. fumigatus conidia and that genetically identical conidia within a population vary in the rate of breaking dormancy and growth. That genetically identical individuals show phenotypic variation that is increased by environmental stress suggests
A. fumigatus might employ a bet-hedging strategy to ensure survival of progeny in varied hostile environments. It seems likely that a similar bet-hedging strategy would also be used by the many fungi that produce large quantities of wind-dispersed spores across varied environments.

\section{Acknowledgments}

The authors thank Julie Nelson at the UGA CTEGD Cytometry Shared Resource Laboratory for assistance with flow analysis.

\section{Funding}

B.C. was supported by National Science Foundation IDEAs grant DGE-1545433. This work was supported by funding from the UGA Department of Plant Biology and the Franklin College of Arts \& Sciences.

\section{Conflicts of interest}

None declared.

\section{Literature cited}

Amich J, Calera JA. 2014. Zinc acquisition: a key aspect in Aspergillus fumigatus virulence. Mycopathologia. 178:379-385.

Araujo R, Rodrigues AG. 2004. Variability of germinative potential among pathogenic species of Aspergillus. J Clin Microbiol. 42:4335-4337.

Bates D, Maechler M, Bolker B, Walker S, Christensen RHB, et al. 2020. lme4: Linear Mixed-Effects Models Using "Eigen" and S4. R Package Version. 1.1-21. https://cran.r-project.org/web/pack ages/lme4/index.html.

Bongomin F, Gago S, Oladele RO, Denning DW. 2017. Global and multi-national prevalence of Fungal diseases-estimate precision. J Fungi (Basel). 3:57. 
Botts MR, Hull CM. 2010. Dueling in the lung: how Cryptococcus spores race the host for survival. Curr Opin Microbiol. 13:437-442.

Brown GD, Denning DW, Gow NA, Levitz SM, Netea MG, et al. 2012. Hidden killers: human fungal infections. Sci Transl Med. 4:165rv113.

Brown W. 1922. On the germination and growth of fungi at various temperatures and in various concentrations of oxygen and of carbon dioxide. Ann Bot. 36:257-283.

Crawley MJ. 2012. Survival Analysis. In: The R Book. West Sussex, UK: John Wiley \& Sons, Ltd. p. 869-892. https://www.amazon. com/R-Book-Michael-J-Crawley/dp/0470973927? asin $=0470973927 \&$ revisionId $=\&$ format $=4 \&$ depth $=1$.

D'Agostino RB, Belanger A, D'Agostino RB. 1990. A suggestion for using powerful and informative tests of normality. Am Stat. 44:316-321.

Dunn OJ. 1964. Multiple comparisons using rank sums. Technometrics. 6:241-252.

Errasquin EL, Patino B, Fernandez RM, Vazquez C. 2002. Occurrence of Aspergillus fumigatus in a compost polluted with heavy metals. Microbiol Comp. 487-494. https://link.springer.com/book/10. 1007/978-3-662-08724-4.

Haas H. 2012. Iron - a key Nexus in the virulence of Aspergillus fumigatus. Front Microbiol. 3:28.

Jackson JC, Higgins LA, Lin X. 2009. Conidiation color mutants of Aspergillus fumigatus are highly pathogenic to the heterologous insect host Galleria mellonella. PLoS One. 4:e4224.

Meletiadis J, Meis JF, Mouton JW, Verweij PE. 2001. Analysis of growth characteristics of Filamentous Fungi in different nutrient media. J Clin Microbiol. 39:478-484.
Loo M. 1976. Some required events in conidial germination of Neurospora crassa. Dev Biol. 54:201-213.

Momany M, Taylor I. 2000. Landmarks in the early duplication cycles of Aspergillus fumigatus and Aspergillus nidulans: polarity, germ tube emergence and septation. Microbiology (Reading). 146 Pt 12: 3279-3284.

Momany M, Westfall PJ, Abramowsky G. 1999. Aspergillus nidulans swo mutants show defects in polarity establishment, polarity maintenance and hyphal morphogenesis. Genetics. 151: 557-567.

Osherov N. 2009. Conidial Germination in Aspergillus fumigatus. In: JPS Latgé, editor. Aspergillus Fumigatus and Aspergillosis. Washington, DC: ASM Press. p. 131-142.

Osherov N, May GS. 2001. The molecular mechanisms of conidial germination. Fems Microbiol Lett. 199:153-160.

Schmit JC, Brody S. 1976. Biochemical Genetics of Neurospora crassa conidial germination. Bacteriol Rev. 40:1-41.

Tepsic K, Gunde-Cimerman N, Frisvad JC. 2006. Growth and mycotoxin production by Aspergillus fumigatus strains isolated from a saltern. Fems Microbiology Letters. 157:9-12.

Wang Z, Miguel-Rojas C, Lopez-Giraldez F, Yarden O, Trail F, et al. 2019. Metabolism and development during conidial germination in response to a Carbon-Nitrogen-Rich Synthetic or a Natural source of nutrition in Neurospora crassa. MBio. 10: e00192-19. 